\title{
Security in Distributed File System
}

\author{
Shwetha K. S., Chandramouli H
}

\begin{abstract}
The investigation of little documents is required to give singular clients the most recent data and improved administrations. Every one of the machines is required to be under a typical director and have the option to impart safely. Huge information is the center subject in enterprises and research fields just as for society overall. Hadoop is the most generally utilized device for huge information examination in internet-based life like Google, Facebook, Yahoo, and Amazon and so on. Hadoop essentially uses Distributed File System for the capacity of an enormous volume of unstructured, ongoing information and streams at a high speed. It has given exact significance to information stockpiling in Hadoop, however, the security of information has overlooked and exceptionally least significance was given. We have algorithms or methodologies proposed.
\end{abstract}

Keywords: Hadoop, Distributed File System, Security, Authentication, Authorization, Encryption

\section{INTRODUCTION}

The fast improvement of computer procedure and system correspondence, database innovation has turned into a significant specialized strategy for sorting out and overseeing monstrous information in current society; so it is the establishment of system data the executive's framework. Then, to join database innovation with system correspondence, man-made consciousness, object-arranged programming, and parallel figuring is the significant normal for database innovation application at present. [1]

In the innovation arranged period of today, there is a developing dissimilarity between the measure of information being produced and the capacity to process and dissect this information. Database the executive's frameworks are intended to oversee such a huge measure of information. The information being created now daily from different sources, for example, interpersonal organization, semantic Web, satellites, observation frameworks, gushing information and bioinformatics system is humongous in sum. Besides, these datasets are exceptionally unstructured and subsequently it is by and large hard to store and deal with this information. These parts of the datasets have made the current database management systems (DBMS) somewhat insufficient and wasteful to be conveyed for the executives of the information being produced. This prompted the introduction of a new database the executive's frameworks that not exclusively are proficient yet, also are particularly effective in putting away, questioning, preparing, breaking down and making the

Revised Manuscript Received on December 15, 2019.

Shwetha K. S., Senior Assistant Professor, Department of Information Science and Engineering, New Horizon College of Engineering, Bangalore, INDIA. Email: shwethaise.nhce@gmail.com

Dr. Chandramouli $\mathbf{H}^{2}$, Professor, Department of Computer Science and Engineering, East Point College of Engineering and Technology, Bangalore, INDIA. Email: hcmcool123@gmail.com

information helpful in an even better advantageous manner.

Today, Hadoop is broadly utilized in numerous ventures as a universally useful stage for conveyed stockpiling and preparing huge informational collections on ware computer groups. Conspicuous Hadoop clients incorporate Yahoo, Facebook, IBM, Twitter, and Adobe. Some outstanding venture merchants have been offering either business Hadoop items or specialized help for Hadoop, including Amazon, Microsoft, Oracle, and expert Hadoop organizations, for example, Cloudera. The Hadoop Distributed File System (HDFS) is the capacity part of the Hadoop structure, which is a dispersed, versatile, and compact document framework, intended to keep running on item equipment. Although it has numerous similitudes with other existing disseminated record frameworks, HDFS is particularly intended to be exceptionally shortcoming tolerant, to give high throughput access to application information, and to manage enormous information documents (ordinary gigabytes to terabytes in size). [9]

Hadoop comprises of the Hadoop Distributed File System (HDFS) to store enormous scale information over petabytes in a bunch domain and the MapReduce structure to help parallel handling dependent on HDFS. HDFS was intended to process a lot of information, records more prominent in size of than tens to hundreds GB are overseen by partitioning into 64MB squares. If there should be an occurrence of putting away little records, if the quantity of little documents expands, the number of squares additionally increments, because every little document (each being tens to several $\mathrm{KB}$ ) is overseen in each square. [16]

Hadoop File System was created utilizing an appropriated document framework structure. It keeps running on production equipment. In contrast to other conveyed frameworks, HDFS is exceptionally deficiency tolerant and planned utilizing ease equipment. Offloading the heap rebalancing assignment is recommended to have capacity hubs by having the capacity hubs balance their heaps precipitously. The information, focus administrators out of sight, virtualizes the assets as indicated by the prerequisites of the client and uncover them as capacity pools, which the clients would themselves be able to use to store documents or information objects. Physically, the asset may range over different servers. In sequence energy is a significant essential for capability frameworks. There has been numerous proposition of putting away information over capacity servers. One advance to give information heartiness is to imitate a note with the end goal that every capability server stores a duplicate of the message. A decentralized eradication code is reasonable for use in a distributed storage system [7] 
The structure of the HDFS is to such an extent that it is utilized to store huge documents however wastefulness lies in putting away an enormous number of little records as a result of high memory consumption and inadmissible right to use cost. A little document is a record whose volume is not exactly the HDFS square size. For instance, 10 million documents, each utilizing a square, would use around 3 gigabytes of memory and in this way, putting away and overseeing an enormous number of little records is a major test to HDFS.

The remainder of the paper is sorted out as follows: Section II exhibits the Related Survey; and finally, Section III presents ends and future headings.

\section{LITERATURE SURVEY}

This journal goes double-blind review process, which means that all the reviewer (s) and author (s) identities covered from the reviewer, and vice versa, right through the review process. All submitted manuscripts are reviewed by three reviewer one from India and rest two from out of the country. There should be proper remarks of the reviewers for the purpose of acceptance/ rejection. There should be minimum 01 to 02 week time window for it.

Zhijian $\mathrm{Yu}$; et al [1] went for the issues in college fixed resource the board, for example, covering speculation and development, and low utilization rate, a college fixed resource database data the executive's framework dependent on a web of things is looked into and created. By taking SQL Server as database stage, this framework consolidates the innovation of web of things with computer technology to apply to informatization the executives of college fixed resources. In the meantime, a systemized, institutionalized and logical fixed resource administration framework is developed, which carries new chance and challenge to the change of college fixed resource the executives.

Ying $\mathrm{Wu}$ et al [2] structure and improvement are to take care of the issue of the framework, utilizing web innovation to oversee client database assets. Furthermore, the expansion of the executives of the database assets to any area has understood the remote administration of database framework, sparing framework upkeep cost, giving accommodation to designers and support staff.

Sejal Samaiya et al [3] examine each continuous application need to store enormous measures of information and procedures this information is required for their fruitful activity. Development of continuous framework causes its applications increasingly complex which to require acquiring degree of information that is the reason it is significant for us to oversee information in a sorted out and deliberate way, so in recent years there came idea of "consolidating" database and ongoing innovation which together called Real-Time database System (RTDBMS). All highlights of ongoing database framework are the same as customary database framework like-Data freedom, Concurrency control, Transactions, Database plans and so on in any case, an RTDBMS has the past obligation of guaranteeing a specific degree of trust in gathering the framework's planning requirements. Execution objective of RTDBMS is very not quite the same as traditional database, essential and most significant execution objectives of RTDBMS's are -

"accuracy criteria" and "pre-suspicion of utilization" though ordinary database fundamentally centers around - "normal reaction time". Assessment of RTDBMS should be possible by ascertaining how frequently exchanges are absent there cutoff times, the expense experienced when exchanges miss there cutoff times, the normal "delay" and "lateness" is additionally Calculated of exchanges when cutoff time is being missed, information transient consistency and information outside consistency ought to likewise be known. Likewise traditional database contrast from continuous database in numerous viewpoints. They have an assortment of objectives to play out the undertaking. Number of criteria to play out the undertaking effectively. Suspicion of use. As the ongoing framework is essentially accentuation on time requirements which are commonly given by the creators of use.

Mingyi Zhang et al [4] proposed new procedures and new highlights of the outstanding burden the executive's offices have been actualized in most business database items. In this paper, we give an efficient investigation of the remaining burden the board in the present DBMSs by creating a scientific classification of the outstanding task at hand administration procedures. We apply the scientific categorization to assess and group existing outstanding tasks at hand administration strategies executed in the business databases and accessible in the ongoing exploration writing. We additionally present the basic standards of the present outstanding burden the executive's innovation for DBMSs, examine open issues and layout some examination openings in this exploration zone.

Cong Liao et al [5] study the issue of information position control inside circulated document frameworks supporting distributed storage. Especially, we consider the open-source Hadoop distributed file system (HDFS) as the fundamental engineering and propose an location-aware cloud storage system, named LAST-HDFS, to help and uphold location-aware storage in HDFS-based groups. What's more, it likewise incorporates a checking framework sent at individual hosts to direct and recognize potential information arrangement infringement because of the presence of pernicious information hubs. We completed a broad trial assessment in a genuine cloud condition that exhibits the adequacy and productivity of our proposed framework.

Metha Wangthammang et al [6] DSePHR is proposed in this work to deal with the encoded PHR information on distributed storage. HBase and Hadoop are used in this work. The goal is to give an API to any PHR framework to transfer/download the scrambled PHR information from distributed storage. The DSePHR settle the Name hub memory issues of HDFS when putting away a great deal of little records by ordering the scrambled PHR information into little and enormous documents. The little documents will be taken care of by HBase mapping that is proposed in this work. The memory utilization and the handling time of the proposed DSePHR are assessed utilizing genuine informational collections gathered from different human services networks. 
M. Nithya et al [7] proposes Load Re-balancing method is utilized for Hadoop Re-Distributed Files System utilizing Distributed Hash Table. The anticipated load re-balancing method will be looked at against a concentrated methodology in a creation framework and a contending conveyed arrangement is introduced in the writing. The capacity hubs are organized as a system dependent on dispersed hash table finding a document lump can allude to a quick key query in DHTs, given that an exceptional handle (or identifier) is doled out to each record piece.

Esraa Alshammari et al [8] propose a structure, which envelops various advances cooperating as another carving technique to recover the most potential bits of records that are tainted by $10 \%$, and to guarantee that the documents are effectively cut.

Wei Dai et al [9] present another imitation arrangement approach for HDFS, which can create copy dispersions that are flawlessly even as well as gathering all HDFS reproduction situation necessities.

S. Suganya et al [10] talk about enormous information is the central theme in ventures and research fields just as for society all in all. Examination of Big Data is a prescient investigation as opposed to the conventional elucidating examination of information. Hadoop is the most broadly utilized apparatus for huge information examination in internet-based life like Google, Facebook, Yahoo, Amazon and so on. Hadoop essentially uses Distributed File System for the capacity of a huge volume of unstructured, constant information and streams at a high speed. It has given exact significance to information stockpiling in Hadoop, however, the security of information has overlooked and exceptionally least significance was given. We have a survey of calculations or strategies recommended.

Yunyue Xie et al [11] with Internet improvement, the information on the planet have had an uncommon increment in the previous decade. Customary IT engineering can't address the issues of sparing and handling information, the development of Cloud Computing tackled the issue. Hadoop is an appropriated preparing programming design that keeps running on the Cloud Computing stage, it can store and process huge information. Hadoop Distributed File System (HDFS) and MapReduce are its two fundamental center segments, which execute dispersed document stockpiling and parallel errand preparing individually. So creators will show, investigation, and assess HDFS dependent on Performance Evaluation Process Algebra (PEPA).

Jyoti Kumari et al [12] present the adaptation to internal failure and copy synchronization among capacity servers (Data-hub) without the impedance of metadata in Hadoop. It utilizes a piece list information structure that holds the data of pertinent copies put away server. At whatever point a customer makes compose solicitation to the record put away on the capacity server, the imitations may wind up conflicting and odds of getting issue increments. To improve the adaptation to non-critical failure and copy synchronization, this paper has utilized another system called Rapid reproduction synchronization. In this work, at whatever point the customer puts compose demand, the one duplicate of imitation will be refreshed first and after that the rest of the reproduction quickly gets update. During read demand, the customer will consistently get the altered information.

Zhuozhao $\mathrm{Li}$ et al [13] direct a complete presentation estimation of various applications on scale-up and scale-out bunches designed with HDFS and a remote record framework (i.e., OFS), separately. We distinguish and study how extraordinary employment qualities (e.g., input information size, the quantity of record peruses/composes, and the measure of calculations) influence the exhibition of various applications on the various stages. Because of the estimation results, we additionally propose an exhibition expectation model to enable clients to choose the best stages that lead to the base idleness. Our assessment utilizing a Facebook remaining task at hand follows shows the viability of our expectation model. This examination is relied upon to give a direction to clients to pick the best stage to run various applications with various qualities in the condition that gives both remote and neighborhood stockpiling, for example, HPC bunch and cloud condition.

A. Aashabegum et al [14] manage the production of a single hub and multinode groups of the Hadoop distributed file system. It is demonstrated in this paper it improves the presentation of the framework during various clients get to the framework. It is likewise demonstrated that the multinode bunch decreases time and builds throughput.

Stathis Maneas et al [15] lead a broad investigation of the Hadoop Distributed File System (HDFS's) code advancement. Our investigation depends on the reports and fix records (patches) accessible from the official Apache issue tracker (JIRA) and our objective was to utilize the whole history of HDFS at the time and the extravagance of the accessible information. The motivation behind our examination is to help engineers in improving the structure of comparable frameworks and actualizing increasingly strong frameworks all in all. As opposed to earlier work, our investigation covers all reports that have been submitted over HDFS's lifetime, instead of an examined subset. Moreover, we incorporate all related fix documents that have been checked by the engineers of the framework and order the main drivers of issues at a better granularity than earlier work, by physically reviewing each of the 3302 reports over the initial nine years, because of a two-level characterization conspire that we created. This enables us to exhibit an alternate point of view of HDFS, including an emphasis on the framework's development after some time, just as a nitty-gritty investigation of attributes that have not been recently examined in detail. These incorporate, for instance, the degree and multifaceted nature of issues as far as the size of the fix that fixes it and number of records it influences, the time it takes before an issue is uncovered, the time it takes to determine an issue and how these shift after some time. Our outcomes demonstrate that bug reports establish the most prevailing sort, having a ceaselessly expanding rate after some time. Also, the general extension and unpredictability of reports and fix documents remain shockingly stable all through HDFS' lifetime, in spite of the critical development the code base encounters after some time.

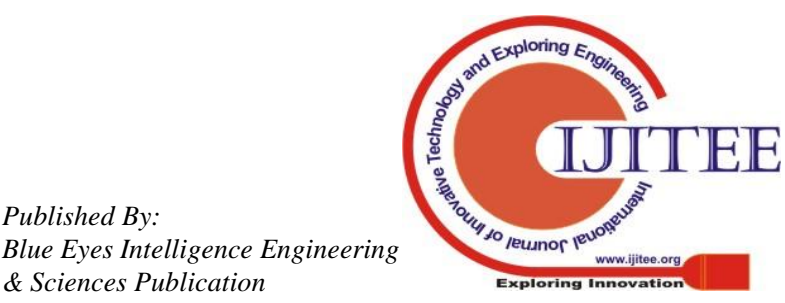


At long last, as a major aspect of our work, we made an itemized database that incorporates all reports and fixes, alongside the key attributes we extricated.

Kyoungsoo Bok et al [16] propose an appropriated store the board plot that considers reserve metadata for effective gets to of little documents in Hadoop Distributed File Systems (HDFS). The proposed plan can decrease the quantity of metadata oversaw by a Name Node since numerous little documents are combined and put away in a lump. It likewise diminishes pointless gets to by keeping the mentioned documents utilizing customers and the reserves of information hubs and by synchronizing the metadata in customer stores as per correspondence cycles.

Lija Mohan et al [17] propose a Balanced MultiFile Input Split (BaMS) system where documents are consolidated and put away. Information is changed over to bytes and all in all, put away in Array Writable configuration. To keep away from the requirement for isolated ordering, we pursue various leveled record naming and putting away plan. The technique portrays how to get to the consolidated records through Map Reduce Programs. Investigation performed on BaMS demonstrates that it is a lot of productive contrasted with the current strategies like HAR and grouping records regarding capacity and access effectiveness.

Zhaowei Li et al [18] dissect the techniques for In-Memory File System utilizing HDFS Lazy Persist procedure and Alluxio to overhaul framework I/O productivity. Furthermore, to keep away from the issue that Lazy Persist methodology should be activated physically each time, we propose HDFS Lazy Persist system programmed trigger component dependent on the insights of information get to data.

J. Jospin Jeya et al [19] talk about a capability framework in the cloud is all around idea out as a most important scale stockpiling framework that has free stockpiling servers. The administration that distributed storage gives is, that can store client's information from remote from side to side system and other verified clients can search out to the information efficiently. Hadoop dispersed document framework is utilized to store vast records consistently and to recuperate those records at high data communication to client applications. Hadoop parts the documents into vast squares and convey them among the hubs in the group. At the point when we recover information from the cloud, it is significant that the calculation and communication overhead have to to be diminished. To diminish the correspondence overhead the server ought to send just the top-n documents dependent on the watchword when the client requests the information records. Since the proprietor need not keep up the duplicate of the documents, it is even more important to make beware of the records accessible and make sure the modernization of the documents put absent in the server from time to time. In HDFS the calculation is done in parallel so the execution time is radically decreased. In the proposed framework for recovering top-n documents, we use Hadoop Distributed File System, with the goal that the hunt time and the correspondence overhead are extraordinarily diminished.

\section{RESULTS AND DISCUSSIONS}

Tharwat El-Sayed et al [20] proposed an upgrade of the Sequence records approach called Small Files Search and Aggregation Node (SFSAN) approach. Our proposed methodology improves the Hadoop execution by defeating a portion of the constraints of the Sequence Files approach and keeping up its focal points.

Table- I: Representative Summary of above Survey

\begin{tabular}{|c|c|}
\hline Reference Paper & Methodology/Description \\
\hline [1] & Fixed Asset Database Information Management System IoT: Barcode technology, Micros oft SQI Server 2008. \\
\hline [2] & $\begin{array}{l}\text { Architecture of the web databas system, web database access techniques is studied. A specific web database res ource } \\
\text { management system integration solution is proposed }\end{array}$ \\
\hline [3] & Real-Time database System (RTDBMS): Real-Time Database Model- Controlling System and Controlled System \\
\hline [4]-Survey & $\begin{array}{l}\text { Workload management techniques into four major technique classes, namely workload characterization, query admission } \\
\text { control, query scheduling and query execution control technique classes. }\end{array}$ \\
\hline [5] & Location-aware cloud storage system, named LAST-HDFS \\
\hline [6] & A distributed storage for storing encrypted PHR data (DSePHR). HBase and Hadoop are used in the proposed DSePHR \\
\hline [7] & Load Rebalancing Algorithm is used for Hadoop Distributed File System using Distributed Hash Table \\
\hline [8] & Carving Technique: art of retrieving files regardless of its type. \\
\hline [9] & Partition Replica Placement Policy (PRPP). \\
\hline [10]-Survey & HDFS review of algorithms or methodologies. (Security) \\
\hline [11] & Model, Analysis, and Evaluate HDFS based on Performance Evaluation Process Algebra (PEPA). \\
\hline [12] & Rapid replica synchronization \\
\hline
\end{tabular}

\section{CONCLUSION}

From the above composition learn around 20 papers mulled over on Distributed File System and we inspected different techniques, calculation and strategies for data security and insurance, structure execution, arrange multifaceted nature of control shows, and so forth... 
Our essential talk and work obsession is on data correspondence on Hadoop Distributed File System. Hadoop Distributed File System is broadly utilized in storage platforms in a clustered architecture with ware equipment. At the point when essential significance was given to information storage, the security of information is ignored. Be that as it may, verifying profoundly private information like Medical, Financial and Social information and so on increases more consideration.

\section{REFERENCES}

1. Zhijian Yu ; Chengyang Yuan ; Ke Zheng "A University Fixed Asset Database Information Management System Based on Internet of Things" 2018 2nd IEEE Advanced Information Management, Communicates, Electronic and Automation Control Conference (IMCEC) Year: 2018 | Conference Paper | Publisher: IEEE.

2. Ying Wu "Design of User Database Resource Management System Based on Web" 2017 International Conference on Computer Technology, Electronics and Communication (ICCTEC) Year: 2017 | Conference Paper | Publisher: IEEE.

3. Sejal Samaiya; Manisha Agarwal Real time database management system 2018 2nd International Conference on Inventive Systems and Control (ICISC) Year: 2018 | Conference Paper | Publisher: IEEE.

4. Mingyi Zhang; Patrick Martin; Wendy Powley; Jianjun Chen "Workload Management in Database Management Systems: A Taxonomy" IEEE Transactions on Knowledge and Data Engineering Year: 2018 | Volume: 30, Issue: 7 | Journal Article | Publisher: IEEE.

5. Cong Liao; Anna Squicciarini; Dan Lin "LAST-HDFS: Location-Aware Storage Technique for Hadoop Distributed File System" 2016 IEEE 9th International Conference on Cloud Computing (CLOUD) Year: 2016 | Conference Paper | Publisher: IEEE.

6. Metha Wangthammang ; Sangsuree Vasupongayya "Distributed storage design for encrypted personal health record data" 2016 8th International Conference on Knowledge and Smart Technology (KST) Year: 2016 | Conference Paper | Publisher: IEEE.

7. M. Nithya ; N. Uma Maheshwari "Load rebalancing for Hadoop Distributed File System using distributed hash table" 2017 International Conference on Intelligent Sustainable Systems (ICISS) Year: 2017 | Conference Paper | Publisher: IEEE.

8. Esraa Alshammari ; Ghazi Al-Naymat; Ali Hadi "A New Technique for File Carving on Hadoop Ecosystem" 2017 International Conference on New Trends in Computing Sciences (ICTCS) Year: 2017|Conference Paper | Publisher: IEEE.

9. Wei Dai ; Ibrahim Ibrahim; Mostafa Bassiouni "A New Replica Placement Policy for Hadoop Distributed File System" 2016 IEEE 2nd International Conference on Big Data Security on Cloud (BigDataSecurity), IEEE International Conference on High Performance and Smart Computing (HPSC), and IEEE International Conference on Intelligent Data and Security (IDS) Year: 2016|Conference Paper | Publisher: IEEE.

10. S. Suganya ; S. Selvamuthukumaran Hadoop "Distributed File System Security -A Review" 2018 International Conference on Current Trends towards Converging Technologies (ICCTCT) Year: 2018 | Conference Paper | Publisher: IEEE.

11. Yunyue Xie; Abobaker Mohammed Qasem Farhan; Meihua Zhou "Performance Analysis of Hadoop Distributed File System Writing File Process" 2018 International Conference on Intelligent Autonomous Systems (ICoIAS) Year: 2018 | Conference Paper | Publisher: IEEE.

12. Jyoti Kumari ; Tarun Biswas; Satyanarayana Vuppala "Enhancing Replica Synchronization in Hadoop Distributed File System" 2018 9th International Conference on Computing, Communication and Networking Technologies (ICCCNT) Year: 2018|Conference Paper | Publisher: IEEE.

13. Zhuozhao Li; Haiying Shen "Measuring Scale-Up and Scale-Out Hadoop with Remote and Local File Systems and Selecting the Best Platform" IEEE Transactions on Parallel and Distributed Systems Year: 2017 | Volume: 28, Issue: 11 | Journal Article | Publisher: IEEE.

14. A. Aashabegum; K. Chitra "Formation of Single and Multinode Clusters in Hadoop Distributed File System" 2017 World Congress on Computing and Communication Technologies (WCCCT) Year: 2017 | Conference Paper | Publisher: IEEE.

15. Stathis Maneas; Bianca Schroeder "The Evolution of the Hadoop Distributed File System" 2018 32nd International Conference on
Advanced Information Networking and Applications Workshops (WAINA) Year: 2018 | Conference Paper | Publisher: IEEE.

16. Kyoungsoo Bok ; Jongtae Lim ; Hyunkyo Oh ; Jaesoo Yoo An efficient cache management scheme for accessing small files in Distributed File Systems 2017 IEEE International Conference on Big Data and Smart Computing (BigComp) Year: 2017|Conference Paper|Publisher: IEEE.

17. Lija Mohan; M. Sudheep Elayidom "Balanced multi file input split (BaMS) technique to solve small file problem in hadoop" 2016 11th International Conference on Industrial and Information Systems (ICIIS) Year: 2016 | Conference Paper | Publisher: IEEE.

18. Zhaowei Li ; Yunlong Yan; Jintao Mo ; Zhaocong Wen; Junmin Wu "Performance Optimization of In-Memory File System in Distributed Storage System" 2017 International Conference on Networking, Architecture, and Storage (NAS) Year: 2017|Conference Paper | Publisher: IEEE.

19. J. Jospin Jeya ; E. Kannan "Enabling top-n file retrieval in cloud storage using hadoop distributed file system" 2016 Second International Conference on Science Technology Engineering and Management (ICONSTEM) Year: $\quad 2016 \mid$ Conference Paper | Publisher: IEEE.

20. Tharwat El-Sayed; Mohammed Badawy; Ayman El-Sayed "SFSAN Approach for Solving the Problem of Small Files in Hadoop" 2018 13th International Conference on Computer Engineering and Systems (ICCES) Year: 2018 | Conference Paper | Publisher: IEEE.

\section{AUTHORS PROFILE}

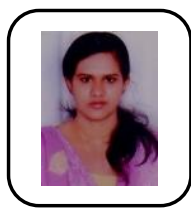

Mrs. Shwetha K S completed M.Tech in the year 2012 and currently doing PH. D under VTU in the field of computer Science and Engineering. She has published many papers in National and International Journals. She holds lifetime ISTE membership. Her research area includes Big data Analytics, Networks.

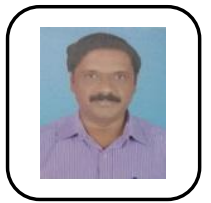

Dr Chandramouli $\mathbf{H}$ received his Ph.D in the year of 2014 and currently working as a Professor in the Department of Computer Science and Engineering at East Point College of Engineering and Technology, Bangalore. He has 22 years of rich experience in the Academics. He has published more than 25 research articles in National and International Journals. He holds CSI membership and an active member in CSI events. His research area includes Wireless sensor network, Resource allocation in Networking, Big Data Analytics. 